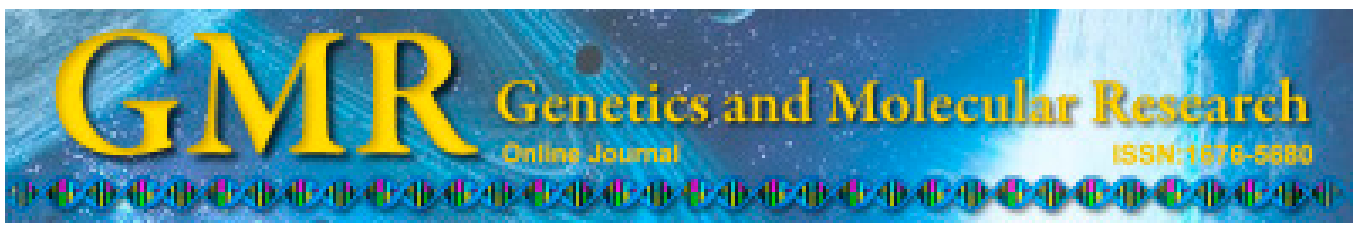

\title{
Human leukocyte antigen-B27 alleles in Xinjiang Uygur patients with ankylosing spondylitis
}

\author{
H.-Y. Zou, W.-Z. Yu, Z. Wang, J. He and M. Jiao \\ Institute of Clinical Medicine, \\ Urumqi General Hospital, Lanzhou Military Area Command, Urumqi, \\ Xinjiang, China
}

Corresponding author: H.-Y. Zou

E-mail: yijiamin2000@yeah.net

Genet. Mol. Res. 14 (2): 5652-5657 (2015)

Received August 19, 2014

Accepted January 13, 2015

Published May 25, 2015

DOI http://dx.doi.org/10.4238/2015.May.25.17

\begin{abstract}
We investigated the distribution of human leukocyte antigen (HLA)-B27 subtypes in Uygur ankylosing spondylitis patients in Xinjiang. B27-positive patients with ankylosing spondylitis were subtyped by using polymerase chain reaction-sequence-based typing. The HLA-B27 subtype frequencies of Uygur patients were compared with those in Han patients in Xinjiang and the other areas of China. B*2705 was the predominant subtype in Uygur patients with a frequency of $58.95 \%$, which was much higher than that in Han patients in Xinjiang $(31.58 \%, \mathrm{P}<0.05)$ and the other areas of China (excluding the Shandong region, which was $63.89 \%)$. The frequency of $\mathrm{B}^{*} 2704(27.37 \%)$ in Uygur patients was the lowest and significantly lower than that in Han patients $(61.18 \%, \mathrm{P}<0.05)$ and in 8 other areas of China. $\mathrm{B} * 2710$ has not been previously reported in Uygur ankylosing spondylitis patients; $B * 2704$ was the main $(61.18 \%)$ subtype in Han patients in Xinjiang, followed by $\mathrm{B} * 2705(31.58 \%)$ and was similar to the characteristics of Han patients in the other areas of China. B*2724 in Han ankylosing
\end{abstract}


spondylitis patients has not been previously reported. Additionally, the $B * 2702 / B * 2705$ homozygote was identified in Uygur patients. $B * 2702 / B * 2704, B * 2704 / B * 2705$, and $B * 2705 / B * 2705$ homozygotes were identified in 3 Han patients. The distribution of HLAB27 subtypes in Uygur ankylosing spondylitis patients in Xinjiang significantly differed from that in Han patients. Understanding the distribution of HLAB27 subtypes in ethnic minority populations of Xinjiang is important for anthropological genetic studies and for analyzing the impact of genetic background on ankylosing spondylitis susceptibility.

Key words: Ankylosing; Human leukocyte antigen; Spondylitis; Uygur nationality

\section{INTRODUCTION}

Human leukocyte antigen (HLA)-B27 is closely associated to ankylosing spondylitis (AS), but the pathogenesis of AS remains unclear. AS may be associated with many factors, including genetic and immune-related factors. The polymorphism level is high in the HLA-B27 allele. As HLA-B27 typing technology has improved, many new subtypes have been identified. The number of HLA-B27 subtypes (HLA-B*2701-2721, 2723-2736) reached 35 in 2007 (Xiao et al., 2008). There may be 1 or more different base sequences among the B27 subtypes, which are mainly focused on exons 2 and 3 (B27 antigen peptide binds to the $\alpha 1$ and $\alpha 2$ structural domains) (Xu et al., 2007). The frequency distribution of B27 subtypes differs in different countries and races (Li et al., 2002). It is particularly important to investigate the relationships between various HLA-B27 subtypes and AS to explain the mechanism of HLA-B27 in AS.

Recently, studies about Han patient HLA-B27 subtypes have been increasingly reported (Cui et al., 2003; Li et al., 2006, 2007; Xiao et al., 2008) in different regions. However, no studies have examined the distribution of B27 subtypes in minorities. In this study, we conducted polymerase chain reaction (PCR)-sequence-based typing (SBT) to analyze the distribution of HLA-B27 subtypes in ankylosing patients in Uygur and Han patients. We investigated the distribution of HLA-B27 subtypes in Uygur AS patients in Xinjiang.

\section{MATERIAL AND METHODS}

\section{General information}

A total of 247 cases of AS from The Rheumatism Immunity branch of each hospital in Urumqi were collected. The Revised Diagnostic Criteria of 1984 in New York was used to confirm the diagnosis of each patient. All patients were positive for HLA-B27 according to PCR-sequencespecific amplification. Ninety-five AS cases in Uygur included 74 males and 21 females, aged 1773 years; the 152 cases of Han patients included 119 male and 33 female cases, aged 17-77 years.

\section{Blood sample collection}

We collected $5 \mathrm{~mL}$ venous blood from each patient, added $10 \%$ ethylenediaminetetraacetic acid as an anticoagulant, and stored the samples at $-20^{\circ} \mathrm{C}$. 


\section{Typing test of HLA-B27}

\section{DNA template preparation}

We used the AxyPrep ${ }^{\mathrm{TM}}$ genomic DNA isolation kit to extract DNA from the peripheral blood according to the manufacturer instructions (Corning, Inc., Corning, NY, USA). The quality of DNA was 1.7-2.0. The concentration of DNA was $20-80 \mathrm{ng} / \mu \mathrm{L}$, and was used for PCR- sequence-specific amplification and PCR-SBT.

\section{PCR-SBT high-resolution genotyping of HLA-B27 alleles}

Exons 2, 3, and 4 of B spots on the genome were amplified through an HLA-B PCR in 1 tube. The amplified positive fragment was used for high-resolution genotyping as a template in the SBT sequence reaction. The sequence was determined using a 3730xl Genetic Analyzer (Applied Biosystems, Foster City, CA, USA), after which we determined the high-resolution genotype of $\mathrm{B}$ spots. We covered 35 high-resolution genotypes of the HLA-B27 allele (HLA-B*2701-2721, 2723-2736), as described by the World Health Organization (WHO).

\section{Lower resolution genotyping of HLA-B27 alleles}

Classical PCR-sequence-specific amplification was conducted according to Xiao et al. (2008). The lower resolution genotype of the HLA-B27 allele accounted for 60.53 and $63.16 \%$, as described by the WHO in 2007 .

\section{Statistical analysis}

All data in this study were analyzed using the PEMS 3.1 statistical software. The gene frequency was determined using the direct count method with the following formula: gene frequency $=$ number of detected gene patients/total number of patients. $\mathrm{P}<0.05$ was considered to be statistically significant.

\section{RESULTS}

\section{Proportion of HLA-B27 allele in AS patients}

Five HLA-B27 allele subtypes were detected in 95 cases of Uygur AS. The distribution frequency was $\mathrm{B} * 2705(58.95 \%, 56 / 95), \mathrm{B} * 2704(27.37 \%, 26 / 95), \mathrm{B} * 2702(6.32 \%$, 6/95), B*2707 (5.26\%, 5/95), and B*2710 (1.05\%, 1/95). The B*2702/B*2705 homozygote was identified in Uygur AS patients. Six HLA-B27 allele subtypes were detected in 152 cases of Han patients. The distribution frequency was $\mathrm{B} * 2704(61.18 \%, 93 / 152), \mathrm{B} * 2705$ (31.58\%, 48/152), B*2707 (1.97\%, 3/152), B*2715 (1.97\%, 3/152), B*2702 (0.66\%, 1/152), B*27024 $(0.66 \%, 1 / 152)$. The $\mathrm{B} * 2702 / \mathrm{B} * 2704, \mathrm{~B} * 2704 / \mathrm{B} * 2705$, and $\mathrm{B} * 2705 / \mathrm{B} * 2705$ homozygotes were identified in 3 Han patients. 


\section{Frequencies of HLA-B27 allele in AS patients in different areas and ethnic groups in China}

B*2705 was the predominant subtype in Uygur patients with a frequency of $58.95 \%$, a value much higher than that in Han patients in Xinjiang $(31.58 \%, \mathrm{P}<0.05)$ and 7 other areas of China (9.83-41.4\%). There was an exception in the Shandong region, $(63.89 \%)$. The frequency of $\mathrm{B} * 2704(27.37 \%)$ in Uygur patients was the lowest, and was significantly lower than that in Han patients $(61.18 \%, \mathrm{P}<0.05)$ and 8 other areas of China $(36.11-90.4 \%)$. B*2704 was the main $(61.18 \%)$ subtype in Han patients in Xinjiang, followed by B*2705 (31.58\%) and was similar to the characteristics of Han patients in other areas of China (except for the Shandong region; B*2705 was not detected in the Hubei region) (Table 1).

\begin{tabular}{|c|c|c|c|c|c|c|c|c|c|c|}
\hline B27 allele & $\begin{array}{c}\text { Xinjiang } \\
\text { Uygurs }\end{array}$ & $\begin{array}{l}\text { Xinjiang } \\
\text { Han race }\end{array}$ & Guangdong & Tangshan & Jiangxi & Shandong & Beijing & Shanghai & Bubei & Chongqing \\
\hline $\mathrm{B} * 2702$ & 6.32 & 0.66 & 1.73 & 0 & 0 & 0 & 0 & 0 & 0 & 0.75 \\
\hline$B * 2704$ & 27.37 & 61.18 & 88.44 & 58.5 & 54.8 & 36.11 & 54.8 & 76.8 & 90.4 & 73.88 \\
\hline$B * 2705$ & 58.95 & 31.58 & 9.83 & 27.7 & 41.4 & 63.89 & 41.4 & 20.3 & 0 & 22.39 \\
\hline$B * 2706$ & 0 & 0 & 0 & 0 & 0 & 0 & 0 & 1.4 & 0 & 0 \\
\hline$B * 2707$ & 5.26 & 1.97 & 0 & 0.9 & 0 & 0 & 0 & 1.4 & 0 & 0.75 \\
\hline$B * 2708$ & 0 & 0 & 0 & 3.6 & 0 & 0 & 0 & 0 & 0 & 0 \\
\hline$B * 2709$ & 0 & 0 & 0 & 0 & 0 & 0 & 0 & 0 & 0 & 0.75 \\
\hline$B * 2710$ & 1.05 & 0 & 0 & 0 & 0 & 0 & 0 & 0 & 0 & 0 \\
\hline$B * 2711$ & 0 & 0 & 0 & 0 & 0 & 0 & 0 & 0 & 4 & 0 \\
\hline$B * 2713$ & 0 & 0 & 0 & 5.4 & 0 & 0 & 0 & 0 & 0 & 0 \\
\hline $\mathrm{B} * 2715$ & 0 & 1.97 & 0 & 0 & 0 & 0 & 0 & 0 & 4.8 & 0 \\
\hline $\mathrm{B} * 2718$ & 0 & 0 & 0 & 0 & 0 & 0 & 0 & 0 & 0 & 0.75 \\
\hline B*2720 & 0 & 0 & 0 & 0 & 0 & 0 & 0 & 0 & 0 & 0.75 \\
\hline$B * 2722$ & 0 & 0 & 0 & 0 & 0 & 0 & 0 & 0 & 0.8 & 0 \\
\hline$B * 2724$ & 0 & 0.66 & 0 & 0 & 0 & 0 & 0 & 0 & 0 & 0 \\
\hline$B * 2702 / 2704$ & 0 & 0.66 & 0 & 0 & 0 & 0 & 0 & 0 & 0 & 0 \\
\hline$B * 2702 / 2705$ & 1.05 & 0 & 0 & 0 & 0 & 0 & 0 & 0 & 0 & 0 \\
\hline B*2704 /2705 & 0 & 0.66 & 0 & 0 & 0 & 0 & 0 & 0 & 0 & 0 \\
\hline$B * 2705 / 2705$ & 0 & 0.66 & 0 & 0 & 0 & 0 & 0 & 0 & 0 & 0 \\
\hline
\end{tabular}

\section{DISCUSSION}

The distribution of HLA-B27 subtypes showed significant differences in different regions, nations, and races, which may be because of different migration routes. $B * 2705$ is the most widely distributed of all HLA-B27 subtypes worldwide, and is thus considered to be the gene predecessor. The different distribution frequency of $B * 2705$ results from mutations, gene translocation, and other mechanisms (Khan, 2000). B*2705 reportedly accounts for approximately $96 \%$ of white patients with AS. B*2705 is the main alleles in the Caucasian population, accounting for approximately $90 \%$ of implicated alleles. B*2704 accounted for approximately $45 \%$. In the Han population, $B * 2704$ and $B * 2705$ were advantageous subtypes of HLA-B27, particularly $\mathrm{B} * 2704$, which is widely distributed with the highest frequency. The distribution frequency of $B^{*} 2704$ showed large differences, ranging from 36.11 to $90.4 \%$. The distribution frequency of $B * 2705$ also showed large differences, ranging from 9.83 to $41.4 \%$ (except for the Shandong region; $B * 2705$ was not detected in the Hubei region). In Thailand, B*2704 accounts for 91\% (Garcia-Peydró et al., 1999) of AS patients who are HLA-B27-positive. 
A large number of studies revealed differences in different HLA-B27 subtypes and the correlation of different subtypes with AS. The subtypes of B*2701, B*2702, B*2704, $\mathrm{B} * 2705, \mathrm{~B} * 2707, \mathrm{~B} * 2708, \mathrm{~B} * 2710, \mathrm{~B} * 2714, \mathrm{~B} * 2715$, and $\mathrm{B} * 2719$ are generally considered to be susceptible genotypes of AS. However, $B * 2703$ in an African population showed a weak relationship with AS. B*2706 and B*2709 in the Thailand population and a population from Sardinia in Italy were negatively correlated with AS, respectively (Ma and Hu, 2006). Other subtypes were present at frequencies that were too low to determine their correlation with AS.

The results of our study indicated that B*2705 was the predominant subtype in Uygur patients, with a frequency of $58.95 \%$, a value much higher than that in Han patients in Xinjiang $(\mathrm{P}<0.05)$ and other areas of China (except the Shandong region). The frequency of B*2705 in Han patients with AS ranged from 9.83 to $41.4 \%$, between the frequencies of Caucasians $(90 \%)$ and Asians (45\%), and the frequency of B*2704 accounted for $27.37 \%$ of Uygur AS patients. This value was the lowest in Uygur patients (36.11-90.4\%). B*2704 was the main (61.18\%) subtype (Li et al., 2006) in Han patients in Xinjiang, followed by B*2705 (31.58\%) and was similar to the characteristics of Han patients in the other areas of China. Our results indicate that $\mathrm{B} * 2705$ is the predominant susceptibility gene in Uygur AS patients in Xinjiang, while B*2704 was the predominant susceptibility gene in Han patients with AS in Xinjiang.

Seven HLA-B27 allele subtypes were detected in 247 cases of AS patients. Five HLAB27 allele subtypes were detected in Uygur AS patients, of B*2710 had not been previously reported in this group. Six HLA-B27 allele subtypes were detected in Han patients, including B*27024, which had not been previously reported. B*2715 was reported in China in 1 other study. Our results also suggested that HLAB27 subtypes in AS patients in Xinjiang showed more polymorphisms than in other areas of China. Seven HLA-B27 allele subtypes were detected in this study, except the $\mathrm{B} * 27024$ subtype, which was detected at too low a frequency to be correlated with disease. In the absence of information regarding the distribution of HLA-B27 subtypes in healthy subjects as a control, the correlation between subtypes B*2702, $\mathrm{B} * 2707, \mathrm{~B} * 2710, \mathrm{~B} * 2715$, and $\mathrm{B} * 27024$ and $\mathrm{AS}$ in this region could not be determined. A $B * 2702 / B^{*} 2705$ homozygote was identified among the Uygur AS patients. B*2702/B*2704, $B * 2704 / B * 2705$, and $B * 2705 / B * 2705$ homozygotes were identified in 3 Han patients. Few studies have examined the relationship between HLA-B27 homozygotes, AS, and AS clinical manifestations.

Xinjiang lies inland in Eurasia and is an important area of communication. It was also an important section of the Silk Road in ancient times. Complicated population migrations have occurred throughout the history of this region. Genetic studies have demonstrated that the Silk Road played a role in gene exchange and gene fusion in Eurasian groups (Yang et al., 2008). This particular historical era may be the primary cause of the difference of the distribution of HLA-B27 subtypes in Uygur AS patients in Xinjiang and Han patients in other areas of China. It also may be the likely primary origin of HLA-B27 subtypes in AS patients in Xinjiang, which showed a higher number of polymorphisms than did other areas of China.

In the Xinjiang region, genetic background is complex, and the mutant gene shows high heterogeneity; the characteristics of genetic epidemiology were significant (Yu et al., 2001). Available survey data showed that the frequency of positive expression of HLA-B27 was markedly different between Uygur patients with AS and Han patients in Xinjiang. There were more Han patients with AS than there were Uygur patients (Mo et al., 2010). The distribution of HLA-B27 subtypes in Uygur AS patients in Xinjiang was significantly different 
from that in Han patients. Examination of the distribution of HLA-B27 subtypes in ethnic minority populations of Xinjiang is very important for anthropological genetic studies and for analyzing the impact of genetic background on AS susceptibility.

\section{ACKNOWLEDGMENTS} \#81172840).

Research supported by the National Natural Science Foundation of China (grant

\section{REFERENCES}

Cui LF, Song HD and Li HF (2003). The correlational research on HLA-B27 allele and AS. Chin. J. Rheumatol. 7: 55-56. Garcia-Peydró M, Marti M and López de Castro JA (1999). High T cell epitope sharing between two HLA-B27 subtypes $(\mathrm{B} * 2705$ and $\mathrm{B} * 2709)$ differentially associated to ankylosing spondylitis. J. Immunol. 163: 2299-2305.

Ma HJ and Hu FP (2006). Diversity of human leukocyte antigen-B27 alleles in Han population of Hunan province, southern China. Tissue Antigens 68: 163-166.

Khan MA (2000b). HLA-B27 polymorphism and association with disease. J. Rheumatol. 27: 1110-1114.

Li QW, Mao YX, Li WC, and Chen LH (2006). Polymorphism analysis of HLA-B27 genes in AS patients in Shandong. Med. Lab. Sci. Clin. 17: 36-37.

Li W, Wu Q and Yu GM (2002). The relationship between HLA-B27 subtypes and AS research progress. Immunology 18: 191-194.

Li W and Huang X (2007). The correlational research on HLA-B27 subtypes and AS. J. Clin. Hematol. 10: 218-220.

Mo HM, Tian YF, Aziguli and Ma HX (2010). Study on the distribution in age and gender in ankylosing spondylitis with HLA-B27 positive in Uygur and Han of Xinjiang. Lab. Med. Clin. 7: 491-492.

Yang LQ, Tan SJ, Yu HJ, Zheng BR, et al. (2008). Polymorphism of mitochondrial DNA reveals gene fusion of the nation living in upper the Silk Road region. J. Life Sci. 38: 368-376.

Yu WZ, Li HJ, Li L, Zhang YH, et al. (2001). The characteristics of genetic epidemiology in China's silk road region. Acta Anthropol. Sin. 20: 69-75.

Xiao LL, Luo M, Guo W, Zhang H, et al. (2008). High resolution alleles of HLA-B27 expression in 1606 suspected cases patients with ankylosing spondylitis. Acad. J. Guangzhou Med. Coll. 36: 5-8.

$\mathrm{Xu} \mathrm{WH}, \mathrm{Fu}$ SM and Zhao Y (2007). Advances in HLA-B27 subtypes and their relationship with ankylosing spondylitis. Mianyixuezazhi 15: 10-12. 